\title{
Metallicity effects in the chemical evolution from AGB stars to $\mathrm{PNe}$
}

\author{
D.A. García-Hernández ${ }^{1}$, P. García-Lario ${ }^{1,2}$, B. Plez $^{3}$, A. Manchado ${ }^{4}$ \\ and F. D'Antona ${ }^{5}$ \\ ${ }^{1}$ ISO Data Centre. European Space Astronomy Centre, Research and Scientific Support \\ Deparment of ESA, Madrid, Spain. \\ email: Anibal.Garcia@sciops.esa.int \\ ${ }^{2}$ Herschel Science Centre. European Space Astronomy Centre, Research and Scientific Support \\ Deparment of ESA, Madrid, Spain \\ ${ }^{3}$ GRAAL, CNRS UMR 5024, Université de Montpellier 2, Montpellier, France \\ ${ }^{4}$ Instituto de Astrofísica de Canarias, La Laguna, Tenerife, Spain \\ ${ }^{5}$ Osservatorio Astronomico di Roma, MontePorzio Catone, Italy
}

\begin{abstract}
We present the main results derived from a chemical abundance analysis carried out on a large sample of massive galactic O-rich AGB stars $\left(\mathrm{M}>3-4 \mathrm{M}_{\odot}\right)$. Combining these results with previous studies made on a similar sample of luminous AGB stars belonging to the Magellanic Clouds we provide strong observational evidences that metallicity effects are playing a more important role than generally assumed in chemical evolution models. This concerns not only the onset of the so-called "hot bottom burning", the efficiency of the third dredge-up and the s-process nucleosynthesis as derived from our optical observations, but also the dust production efficiency and the chemical properties of the dust grains in the shell, as inferred from the available infrared data. We find $\mathrm{Li}$ overabundances in the galactic stars studied, indicating that they are actually "hot bottom burning" AGB stars. Similar Li overabundances are also observed in the most luminous Magellanic Cloud AGB stars. However, the AGB stars in our galactic sample are not enriched in $\mathrm{Zr}$, in contrast to what is observed in the Magellanic Clouds. In addition, many stars in the galactic sample appear heavily obscured in the optical, suggesting a much more efficient dust production and/or stronger mass loss rates which eventually can be translated into shorter AGB lifetimes.
\end{abstract}

Keywords. stars: AGB and post-AGB-stars: abundances-nuclear reactions, nucleosynthesis

\section{Introduction}

The Asymptotic Giant Branch (AGB) is formed by stars with initial masses in the range between 0.8 and $8 \mathrm{M}_{\odot}$ in a late stage of their evolution. The internal structure of an AGB star consists of an electron-degenerate C-O core surrounded by a shell of He. During most of the time $\mathrm{H}$ burning is the main source of energy for the AGB star but, occasionally, the inner He shell ignites in a thermal pulse and, eventually, the byproducts of He burning may reach the outer layers of the atmosphere in a dredge-up of processed material (the so-called $3^{r d}$ dredge-up). Repeated thermal pulses add carbon to the stellar surface. As a consequence, AGB stars originally O-rich can turn into C-rich AGB stars $(\mathrm{C} / \mathrm{O}>1)$ after a few thermal pulses. This would explain the evolutionary sequence M-MS-S-SC-C observed in AGB stars. However, this does not apply to the case of the more massive AGB stars $\left(\mathrm{M} \gtrsim 3-4 \mathrm{M}_{\odot}\right)$, where the convective envelope can penetrate the H-burning shell activating the so-called "hot bottom burning" (hereafter, HBB). This is because HBB prevents the formation of carbon, preserving the original O-rich composition of the stellar atmosphere throughout the whole AGB evolution. HBB models (e.g. Mazzitelli et al. 1999, hereafter MDV99) predict also the production of short-lived 
${ }^{7} \mathrm{Li}$ which should be detectable, at least for some time, on the stellar surface. The HBB activation in massive O-rich AGB stars is supported by studies of AGB stars in the Magellanic Clouds (hereafter, MCs) (e.g. Plez et al. 1993). These stars are actually very luminous $\left(-7 \leqslant \mathrm{M}_{\text {bol }} \leqslant-6\right)$ Li-rich stars. In our own Galaxy, only a handful of Li-rich stars have been found so far (e.g. Abia et al. 1993) and, unlike those detected in the MCs, they are not so luminous $\left(-6 \leqslant \mathrm{M}_{b o l} \leqslant-3.5\right)$. Moreover, they are relatively low-mass $\left(\mathrm{M} \lesssim 2-3 \mathrm{M}_{\odot}\right)$ AGB stars with $\mathrm{S}-, \mathrm{SC}$ - and $\mathrm{C}$-spectral types where HBB is not expected to be active.

Another important characteristic of AGB stars is the presence of neutron-rich elements (like Sr, Y, Zr, Ba, La, Nd, Tc, etc.) in their atmospheres which are the consequence of the slow-neutron captures produced during the thermally pulsing AGB phase. These s-process elements are believed to form during the interpulse period through the ${ }^{13} \mathrm{C}(\alpha, \mathrm{n}){ }^{16} \mathrm{O}$ reaction. ${ }^{22} \mathrm{Ne}$ is another neutron source, but only becomes active at higher temperatures, during the thermal pulses. An interesting consequence from the observational point of view is that a different s-element pattern is expected depending on which neutron source is active. According to the most recent models ${ }^{13} \mathrm{C}$ is the preferred neutron source for masses around 1-3 $\mathrm{M}_{\odot}$ while for intermediate mass stars $\left(\mathrm{M} \gtrsim 3-4 \mathrm{M}_{\odot}\right)$ neutrons are thought to be mainly released by the ${ }^{22} \mathrm{Ne}(\alpha, \mathrm{n}){ }^{25} \mathrm{Mg}$ chain (see e.g. Busso et al. 1999). In the literature, there is strong evidence that most galactic AGB stars enriched in sprocess elements have masses around $1-3 \mathrm{M}_{\odot}$ (e.g. Abia et al. 2001). Unfortunately, a confrontation of the predictions made by the $\mathrm{HBB}$ and s-process nucleosynthesis models with observations of more massive AGB stars in our Galaxy is not yet available.

\section{Observations}

The best galactic candidates to study the activation of HBB and s-process nucleosynthesis in massive AGB stars are the so-called $O H / I R$ stars. These are luminous O-rich AGB stars which are extremely bright in the infrared, showing a characteristic doublepeaked $\mathrm{OH}$ maser emission at $1612 \mathrm{MHz}$. These stars are also known to be very long period variables (LPVs), sometimes with periods of more than 500 days and large amplitudes of up to 2 bolometric magnitudes. However, they experience very strong mass loss rates (up to several times $10^{-5} \mathrm{M}_{\odot} \mathrm{yr}^{-1}$ ) and most of them are usually heavily obscured by thick circumstellar envelopes, making optical observations very difficult. Thus, a large sample (102) of long-period (300-1000 days), large amplitude variability (up to 8-10 magnitudes in the $\mathrm{V}$ band), late-type (> M5) O-rich AGB stars displaying $\mathrm{OH}$ maser emission with a wide range of expansion velocities $\left(\sim 1-20 \mathrm{~km} \mathrm{~s}^{-1}\right)$ was carefully selected for analysis. These stars are expected to be massive stars in the final stages of their AGB evolution. Consistently, they display strong IR excesses, which were detected by IRAS.

High resolution optical echelle spectra $(\mathrm{R} \sim 40,000-50,000)$ of these stars were obtained during several observing periods in 1996-1997. Because of the very red colours of the sources observed, the $\mathrm{S} / \mathrm{N}$ ratios achieved in the reduced spectra can strongly vary from the blue to the red orders $(\sim 10$ at $\sim 6000 \AA$ while $>100$ at $\sim 8000 \AA)$. Here we report on the main results obtained.

\section{Chemical abundances}

We detected the presence of the $\mathrm{Li}$ I resonance line at $6708 \AA$ in 25 of the sources in the sample with a wide variety of strengths, while we did not find any signature of this line in 32 of the stars. The remaining 45 stars were too red or simply the optical counterpart was not found at the moment of the observations, heavily obscured by their 


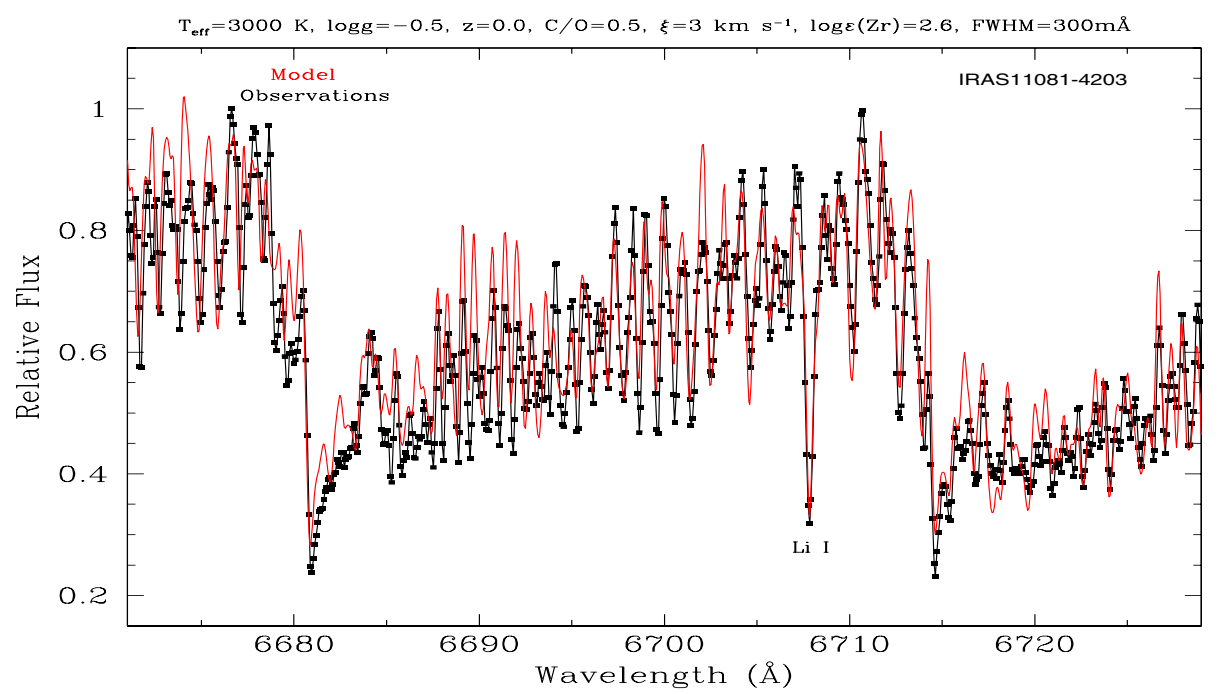

Figure 1. Best model fit and observed spectrum in the region $6670-6730 \AA$ for the star IRAS 11081-4203. The $T_{\text {eff }}$ and $\mathrm{Li}$ abundance derived from this spectrum was $3000 \mathrm{~K}$ and $\log \varepsilon(L i)=$ +1.3 , respectively. The parameters of the best model atmosphere fit are indicated in the top label.

thick circumstellar envelopes. Interestingly, the bandheads of $\mathrm{ZrO}$ (i.e. those at 6474 and $6495 \AA$ ) seem to be absent in all spectra. These $\mathrm{ZrO}$ bandheads (as well as those corresponding to other s-element oxides such as $\mathrm{LaO}$ or $\mathrm{YO}$ ) are very strong in galactic S-stars and in massive MC AGB stars. Our sample stars also display strong Rb I and K I lines at $\sim 7800$ and $7699 \AA$, respectively. However, the latter resonance lines usually show complex profiles and blue-shifted circumstellar components.

We carried out a chemical abundance analysis by combining state-of-the-art line blanketed model atmospheres and synthetic spectroscopy with extensive linelists. Then we derived the Li and $\mathrm{Zr}$ abundances in those stars for which an optical spectrum could be obtained (see García-Hernández et al. 2006a for details about the technique used). As an example, the best fit in the $6670-6730 \AA$ spectral region around the Li I line is presented in Figure 1 for the star IRAS 11081-4203. The elemental abundance of Rb in these stars is also of special importance because it can be used as an indicator of the neutron density at the s-process element formation site and as a signature of the operation of the ${ }^{22} \mathrm{Ne}$ neutron source in these stars. The detailed analysis of the $\mathrm{Rb}$ abundances will be presented in a forthcoming paper (García-Hernández et al. 2006b).

Our chemical abundance analysis shows that half of the stars analysed show Li overabundances in the range $\log \varepsilon(\mathrm{Li}) \sim 0.5-3.0 \pm 0.4-0.6$ dex. The $\mathrm{Li}$ overabundances observed are interpreted as a signature of the activation of $\mathrm{HBB}$, confirming that they are indeed massive AGB stars ( $\mathrm{M} \gtrsim 4 \mathrm{M}_{\odot}$ according to MDV99 HBB models). The nondetection of the $\mathrm{ZrO}$ molecular bands at $6474 \AA$ and $6495 \AA$ in any of the stars analysed imposes severe upper limits to the zirconium abundance $\left([\mathrm{Zr} / \mathrm{Fe}]<0.0-0.25\right.$ for $\mathrm{T}_{\text {eff }} \geqslant$ $3000 \mathrm{~K}$ and $[\mathrm{Zr} / \mathrm{Fe}]<0.25-0.50$ for $\left.\mathrm{T}_{\text {eff }}<3000 \mathrm{~K}\right)$. If the $\mathrm{Zr}$ enhancement is taken as a representative for the s-process enrichment, our results indicate that massive AGB stars in our Galaxy are not s-process enriched. They also show the first observational evidence of the operation of ${ }^{22} \mathrm{Ne}$ as the dominant neutron source in these HBB AGB stars, as inferred from the strong Rb overabundances found (García-Hernández et al. 2006b). 
Table 1. Main observational properties of Galactic HBB AGB stars compared to those of Magellanic Cloud HBB AGB stars. Differences are attributed to metallicity effects.

\begin{tabular}{lcccccc}
\hline & $\begin{array}{c}\text { Dust } \\
\text { production }\end{array}$ & Dredge-up & lifetime & $\begin{array}{c}\text { HBB } \\
\text { activation }\end{array}$ & {$[\mathrm{s} / \mathrm{Fe}]$} & $\begin{array}{c}\text { Neutron } \\
\text { source }\end{array}$ \\
\hline $\begin{array}{l}\text { Galaxy } \\
\text { MCs }\end{array}$ & $\begin{array}{c}\text { efficient } \\
\text { non-efficient }\end{array}$ & $\begin{array}{c}\text { non-efficient } \\
\text { efficient }\end{array}$ & $\begin{array}{c}\text { small \# of TPs } \\
\text { large \# for } \mathrm{M}>4 \mathrm{M}_{\odot}\end{array}$ & $<0.5 \mathrm{dex}$ & ${ }^{22} \mathrm{Ne}$ \\
\hline
\end{tabular}

\section{Comparison with the Magellanic Clouds: metallicity effects}

The situation is quite different for the massive O-rich AGB stars in the MCs. Although $\sim 80 \%$ of them are also Li-rich, indicating that, indeed, they are HBB stars, these stars are s-element enriched, in contrast with the results found in our galactic sample.

We suggest that MC HBB stars are efficiently enriched in s-process elements during their AGB evolution just as a consequence of their lower metallicity. Indeed, theoretical models predict a higher efficiency of the third dredge-up in low metallicity environments (Herwig 2004) with respect to those with solar metallicity (Lugaro et al. 2003). Second, there is an increasing observational evidence that lower metallicity environments are also less favourable to dust production (Sloan et al. 2006). Mass loss, if driven by radiation pressure on the dust grains, might be less efficient with decreasing metallicity (Willson 2000). In that case, longer AGB lifetimes would be expected, which would explain why even the more massive AGB stars in the MCs show a strong s-process enrichment, in contrast to their galactic counterparts. In addition, the lower critical mass needed to develop HBB (e.g. M $>3 \mathrm{M}_{\odot}$ at the metallicity of the LMC, compared to the $\sim 4 \mathrm{M}_{\odot}$ limit in our Galaxy) would favour the simultaneous detection of s-process elements and Li enrichment in a larger number of AGB stars in the MCs, as it is actually observed. Finally, the s-element abundance pattern found in the HBB AGBs in the MCs is in good agreement with the ${ }^{13} \mathrm{C}$ neutron source model predictions at low metallicities (see e.g. Plez et al. 1993). In contrast, our observations show that in the HBB AGB stars in our Galaxy the ${ }^{22} \mathrm{Ne}$ neutron source must be activated (García-Hernández et al. 2006b). In Table 1 we summarize the main differences observed between HBB AGB stars in our Galaxy and those belonging to the MCs, which we attribute to metallicity effects.

\section{References}

Abia, C., Boffin, H. M. J., Isern, J., \& Rebolo, R. 1993, A\& A, 272, 455

Abia, C., Busso, M., Gallino, R., et al. 2001, ApJ, 559, 1117

Busso, M., Gallino, R., \& Wasserburg, G. J. 1999, ARA\&A 37, 239

García-Hernández, D. A., García-Lario, P., Plez, B., et al. 2006a, A\&A, in press (astro-ph/ 0609106)

García-Hernández, D. A., Plez, B., D’Antona, F., et al. 2006b, (in preparation)

Herwig, F. 2004, ApJ, 605, 425

Lugaro, M., Herwig, F., Lattanzio, J. C., et al. 2003, ApJ, 586, 1305

Mazzitelli, I., D’Antona, F., \& Ventura, P. 1999, AछA A, 348, 846

Plez, B., Smith, V. V., Lambert, D. L. 1993, ApJ, 418, 812

Sloan, G. C., Kraemer, K. E., Matsuura, M., et al. 2006, ApJ, 645, 1118

Willson, L.A. 2000, ARA\&A, 38, 573 